\title{
Changes in fatty acid levels (saturated, monounsaturated and polyunsaturated) during pregnancy
}

\author{
Estefania Aparicio ${ }^{1,2 \dagger}$, Carla Martín-Grau ${ }^{1,3 \dagger}$, Carmen Hernández-Martinez ${ }^{1,2,4}$, Nuria Voltas ${ }^{1,2,4}$, \\ Josefa Canals ${ }^{1,2,4}$ and Victoria Arija ${ }^{1,2^{*}}$
}

\begin{abstract}
Background: During pregnancy a high amount of fatty acids (FA) is necessary to meet foetus demands, which vary during gestation. The present study describes the changes in maternal fatty acid concentrations during pregnancy in a sample of pregnant women.

Methods: This is a longitudinal study of 479 pregnant women who were monitored from the first trimester to third trimester of pregnancy. Data on maternal characteristics were recorded and a serum sample was collected in each trimester. The fatty acid profile (saturated (SFA: total, lauric acid, myristic acid, palmitic acid, stearic acid), monounsaturated (MUFA: total, palmitoleic acid, oleic acid) and polyunsaturated fatty acids (PUFA: total omega-6 (n-6), linoleic acid, dihomo- $\gamma$-linolenic acid, arachidonic acid (AA), total omega-3 ( $n-3)$, eicosapentaenoic acid (EPA), docosahexaenoic acid (DHA)) was analysed with a gas chromatography-mass spectrometry combination.

Results: From the first trimester to third trimester of pregnancy, a significant increase in total SFA, total MUFA and total n-6 PUFA was found. ( $p<0.001)$. Nevertheless, the serum concentration of arachidonic acid (AA), eicosapentaenoic acid (EPA) and total n-3 PUFA decreased during gestation $(p<0.001)$. A statistically non-significant result was observed for the docosahexaenoic acid (DHA) serum concentration between the first and third trimesters of pregnancy. Significant correlations were observed between each total fatty acid concentrations of the first and third trimesters.

Conclusion: The circulating serum concentration of SFA, MUFA and n-6 PUFA increases during pregnancy, whereas essential fatty acids such as AA and EPA decrease, and DHA remains unchanged. Further research is necessary to understand the role played by FA throughout gestation.
\end{abstract}

Keywords: Pregnancy, Fatty acid status, DHA, EPA, Omega-3, Omega-6

\section{Introduction}

A good nutritional status of fatty acids (FA) during pregnancy is important for the mother's health in order to meet the needs of foetal growth and development.

\footnotetext{
*Correspondence: victoria.arija@urv.cat

†Estefania Aparicio and Carla Martín-Grau contributed equally to this work.

${ }^{2}$ Institut d'Investigació Sanitària Pere Virgili (IISPV), 43003 Tarragona, Spain Full list of author information is available at the end of the article
}

Studies evaluating the effects of FA deficiency states during pregnancy have mainly focused on polyunsaturated fatty acids (PUFA). In particular, a maternal deficiency of omega-3 polyunsaturated fatty acids ( $\mathrm{n}-3$ PUFA) may affect placental angiogenesis and vasculogenesis, thereby affecting foetal development (brain, muscle, eye, motor nerve and adiposity) [1]. It is known that eicosapentaenoic acid (EPA) and docosahexaenoic acid (DHA) are two of the most important FA for the cognitive and visual 
development of the foetus [2-5]. Maternal fatty acid status is also crucial in late pregnancy because this is when the human foetal brain grows rapidly and high amounts of DHA accumulates in it $[1,5]$. Thus, the foetus during the third trimester is especially vulnerable to developmental deficits due to a poor status of n-3 PUFA, and this could cause problems in neurological development $[6,7]$. However, few studies have assessed the changes in FA during pregnancy [8-10]. An appropriate balance with $n-3$ and omega- 6 polyunsaturated fatty acids ( $n-6$ PUFA) is necessary $[11,12]$ because a high concentration of maternal arachidonic acid (AA) has been associated with deleterious outcomes, such as major depression in the mother [13, 14], or preterm delivery [15]. However, a higher maternal AA/DHA ratio at delivery and heterozygosity for the FADS1 genetic variant have shown positive long-term effects on processing speed in children at 9 years of age [7].

Therefore, an adequate fatty acid profile in early and late pregnancy is crucial for the health of both the mother and the child. To our knowledge there is a dearth of literature on the changes in plasma FA concentrations throughout pregnancy. A recent systematic review analysed how the $n-3$ and n- 6 PUFA profile is modified during pregnancy [16]. It included only three studies [17-19] and showed that DHA and n-6 PUFA concentrations increase throughout pregnancy, while EPA remains unchanged. Similar findings were obtained in research conducted with a Canadian pregnant population with and without gestational diabetes [20]. Saturated fatty acids (SFA) and monounsaturated fatty acids (MUFA) have been studied less $[17,19,21]$. The survey by Pinto et al. [17] of 225 pregnant Brazilian women found an increase in SFA and MUFA concentrations throughout pregnancy. Taking into account the importance of FA status for maternal and foetal health, a better understanding and knowledge of this subject would help obstetrics and health professionals to identify possible abnormal values and decide whether treatment is necessary and which treatment to recommend. The aim of the current study is therefore to analyse the changes in maternal fatty acid concentrations (saturated, monounsaturated and polyunsaturated) during pregnancy in a cohort of pregnant women from a Mediterranean country in Europe.

\section{Material and methods}

\section{Study design and population}

This study researched a longitudinal cohort of pregnant women from the ECLIPSES study [22]. The participants were contacted during the first prenatal visit to one of twelve sexual and reproductive health care services (ASSIR) of the Catalan Institute of Health (ICS) in Tarragona, Spain. The pregnant women were monitored from the first trimester (12th gestational week) to the third trimester (36th gestational week) of pregnancy. The inclusion criteria were: healthy woman older than 18 years of age, pregnancy at $\leq 12$ weeks, ability to understand the local and official state languages and the characteristics of the study, and signing the informed consent form. The women were excluded if they fulfilled any of the following exclusion criteria: multiple pregnancy, having taken iron supplements during the months previous to week 12 of pregnancy, hypersensitivity to egg protein, previous severe disease (immunosuppression) or any chronic disease which could affect their nutritional development (cancer, diabetes, malabsorption, or liver disease).

The present study is a secondary blood sample analysis of a randomized controlled trial of iron supplementation during pregnancy, called the ECLIPSES study. Finally, in the ECLIPSES study, 791 women were recruited at week 12 of pregnancy, and 534 women completed the data on week 36 of pregnancy. The causes of dropout included leaving voluntarily, miscarriage, the emergence of exclusion criteria during pregnancy (such as serious illness that could affect the nutritional status, for instance cancer, diabetes, malabsorption or liver disease) and lost to follow-up. More information can be found in the previous paper [23].

This study was approved by the Clinical Research Ethics Committee of the Jordi Gol Institute for Primary Care Research (IDIAP) and the Pere Virgili Institute for Health Research (IISPV). All participants who agreed to participate signed the informed consent form.

\section{Data collected}

Midwives collected the participants' medical and obstetric history, socioeconomic information, lifestyle habits and anthropometric measurements in the first trimester of pregnancy. The medical and socioeconomic data included maternal age, ethnicity, education level (primary, secondary, or university studies), smoking habits and planned pregnancy, clinical and obstetric history. The socioeconomic level was classified as low, middle or high according to the Catalan classification of occupations (CCO-2011) [24]. Anthropometric measurements were obtained such as maternal height $(\mathrm{cm})$ and weight $(\mathrm{Kg})$, and BMI was calculated. The BMI was categorized as normal weight $\left(\mathrm{BMI}<25 \mathrm{~kg} / \mathrm{m}^{2}\right.$ ) or excess weight (BMI $\geq 25 \mathrm{~kg} / \mathrm{m}^{2}$ ) according to the World Health Organization (WHO) criteria [25].

\section{Blood sampling and determination of the fatty acid profile in plasma}

Blood samples were collected at the first and third trimesters of pregnancy. They were collected in 1 tube of $7.5 \mathrm{ml}$ without anticoagulant and were not mixed for 
$30 \mathrm{~min}$ at room temperature to allow coagulation. The serum was centrifuged and distributed in aliquots of $500 \mu \mathrm{l}$ and stored at $-80^{\circ} \mathrm{C}$. The samples were stored in the BioBank. At the end of the clinical study, samples were thawed and processed simultaneously in order to minimize inter-batch variation [22].

FA such as saturated, mono- and polyunsaturated FA were analysed. A combination of gas chromatography-mass spectrometry (GC-MS) was used to analyse FA using 7890A GC equipment coupled to QqQ 7000 Series $^{\circledR}$ (Agilent Technologies Inc., Santa Clara, USA) after their derivatization to methyl ester (FAMEs) due to their higher volatility [26]. A volume of $50 \mu \mathrm{l}$ of plasma samples was blended with internal standard (IS) solution (Myristic d-27 acid, Merck KGaA, Darmstadt, Germany), chloroform and methanolic hydrochloric acid and incubated at $80^{\circ} \mathrm{C}$ during 2 hours. Then, FAMEs were extracted by liquid-liquid extraction by means of hexane and then injected in the GC-MS system. Chromatographic analysis was carried out according to David et al. [26] to determine the 37 FAMEs included in the Food Industry FAME Mix (Restek Corporation, Pennsylvania, USA). FAMEs were split up in a high-polarity column $(100 \mathrm{~m} \times 250 \mu \mathrm{m} \times 0.25 \mu \mathrm{m})(\mathrm{HP}-88$ column, Agilent Technologies Inc., USA) with a temperature program between 140 and $240^{\circ} \mathrm{C}$ at $1 \mathrm{~mL} / \mathrm{min}$ by a carrier gas (helium). Ionization was performed by electronic impart $(70 \mathrm{eV})$ and the mass analyser operated on Selected Ion Monitoring mode (SIM). The processing of the FA sample was described in the previous paper [27]. The FA identified were: SFA, lauric acid, myristic acid, palmitic acid and stearic acid (the sum total of these was calculated to obtain total SFA); MUFA, palmitoleic acid and oleic acid (the sum of these FA was calculated to obtain total MUFA); n-6 PUFA, linoleic acid (LA), dihomo- $\gamma$ linolenic acid and AA (the sum total of these was calculated to obtain total n-6 PUFA); n-3 PUFA, EPA and DHA (the sum of these FA was calculated to obtain total n-3 PUFA). Moreover, total long chain fatty acids was calculated as total SFA + total MUFA + total n-6 PUFA + total n-3 MUFA.

\section{Statistical analysis}

The ability of the study sample to detect differences in FA concentration between the first and third trimesters of pregnancy was calculated using the data from this study in accordance with the following parameters: an alpha risk of 0.05 and a beta risk of 0.20 in a bilateral contrast to paired samples. For total SFA, 444 participants were sufficient to detect a difference equal to or greater than $612 \mu \mathrm{mol} / \mathrm{L}$ considering a standard deviation of $4601.2 \mu \mathrm{mol} / \mathrm{L}$ (mean of $5860,5 \mu \mathrm{mol} / \mathrm{L}$ ). For total MUFA, 440 participants were sufficient to detect a difference equal to or greater than $198 \mu \mathrm{mol} / \mathrm{L}$ considering a standard deviation of $1481.8 \mu \mathrm{mol} / \mathrm{L}$ (mean of $1294.7 \mu \mathrm{mol} / \mathrm{L}$ ). For total n-6 PUFA, 443 participants were sufficient to detect a difference equal to or greater than $403 \mu \mathrm{mol} / \mathrm{L}$ considering a standard deviation of $3026.1 \mu \mathrm{mol} / \mathrm{L}$ (mean of $2844.3 \mu \mathrm{mol} / \mathrm{L}$ ). For total $\mathrm{n}-3$ PUFA, 454 participants were sufficient to detect a difference equal to or greater than $13.5 \mu \mathrm{mol} / \mathrm{L}$ considering a standard deviation of $102.7 \mu \mathrm{mol} / \mathrm{L}$ (mean of $-16.5 \mu \mathrm{mol} / \mathrm{L}$ ). Therefore, our sample showed enough statistical power to detect differences.

For the FA concentration, outlier values were identified by z-score analysis [28], considering an absolute value of \pm 3.29 as the standard value to detect outliers when the sample size is $>100$. Thus, an outlier case was considered when the $\mathrm{z}$-score was above \pm 3.29 or below \pm 3.29 [29]. The results were presented as mean \pm standard deviation (SD) or percentage. The means between groups (the first and third trimesters of pregnancy) were compared by the paired student's t-test. The Pearson correlation was used to assess the association between each FA concentration total at the first and third trimesters. The percentage of variation from the first to third trimester was calculated for each individual FA with the following equation: (final value - initial value)/initial value*100. Statistical analyses were run by SPSS version 25.0 for Windows (SPSS, Chicago, IL, USA). The significance level was at $p$-value $<0.05$.

\section{Results}

\section{Participants' characteristics}

The biochemical profiles of the FA in the first and third trimesters were analysed in 479 pregnant women. The baseline characteristics of pregnant women are shown in Table 1. The maternal age was $30.6 \pm 5.01$ years, and up to $80 \%$ were Caucasian. About $38.3 \%$ of women had a medium educational level and $69.6 \%$ were middle-class. Likewise, 25.3 and $12.5 \%$ of the pregnant women in the sample were overweight or obese, respectively, and $15.3 \%$ of participants reported that they smoked at the beginning of pregnancy. Some variables of baseline characteristics showed missing values, which range from 0.2 to $8.7 \%$.

The FA detected in maternal serum and their concentration $(\mu \mathrm{mol} / \mathrm{L})$ during the first and third trimesters of pregnancy are shown in Table 2. From the first trimester to the third trimester of pregnancy there was a significant increase in total SFA (and their individual FA) and total MUFA (as well as palmitoleic acid and oleic acid) $(p<0.001)$. The percentage of variation of total SFA and total MUFA was $101.02 \pm 93.05$ and $184.93 \pm 154.49$, respectively. In addition, a significant increase in total n-6 PUFA in the third trimester $(p<0.0001)$ was observed 
Table 1 Baseline characteristics of pregnant women in the first trimester

\begin{tabular}{|c|c|}
\hline General characteristics & $\begin{array}{l}\text { Mean } \pm S D^{a} \text { or } \% \\
(n=479)\end{array}$ \\
\hline Maternal age (years) ${ }^{a}$ & $30.6 \pm 5.01$ \\
\hline Parity (yes \%) & 58.8 \\
\hline Planned pregnancy (yes \%) & 80.7 \\
\hline \multicolumn{2}{|l|}{ Maternal ethnic origin (\%) } \\
\hline Caucasian & 81.9 \\
\hline Asian & 0.7 \\
\hline Arab & 7.8 \\
\hline Black & 2.1 \\
\hline Latin American & 7.6 \\
\hline \multicolumn{2}{|l|}{ Maternal educational level (\%) } \\
\hline Low (primary or less) & 30.1 \\
\hline Medium (secondary) & 38.3 \\
\hline High (university or more) & 31.6 \\
\hline \multicolumn{2}{|l|}{ Socioeconomic status (\%) } \\
\hline Low & 13.5 \\
\hline Middle & 69.6 \\
\hline High & 16.9 \\
\hline \multicolumn{2}{|l|}{ BMI $\left(\mathrm{kg} / \mathrm{m}^{2}\right)$ at first trimester (\%) } \\
\hline$<25$ & 62.2 \\
\hline $25-30$ & 25.3 \\
\hline$\geq 30$ & 12.5 \\
\hline \multicolumn{2}{|l|}{ Smoking status (\%) } \\
\hline Smoker & 15.3 \\
\hline Non-Smoker or Ex-Smoker & 84.7 \\
\hline
\end{tabular}

${ }^{\mathrm{a}}$ Mean \pm standard deviation. $B M \mathrm{l}$ body mass index

when it was compared to the maternal FA concentration in the first trimester of pregnancy, with a percentage of $77.85 \pm 85.91$ of variation. Nevertheless, the percentage of variation of AA was $-6.33 \pm 34.88$ with a significant decrease in its concentration from the first to third trimesters. Regarding n-3 PUFA, the EPA concentration decreased during gestation $(p<0.001)$ with a percentage of variation of $-14.93 \pm 68.21$. A statistically non-significant result was observed in the DHA concentration $(p=0.357)$ between the first and third trimesters of pregnancy.

Moreover, as the original study was a clinical trial with iron supplementation, we analysed whether the study group had any effect on the FA concentration. There were no significant differences in FA concentrations between the study groups in the first trimester or in the second trimester.

Moreover, Fig. 1 depicts the correlation of serum concentration of total SFA, MUFA, n-6 PUFA, n-3 PUFA and total FA between the first trimester and third trimester. A significantly low-moderate correlation of each total
FA concentration was observed from the first to third trimesters.

\section{Discussion}

This longitudinal study describes the changes in FA during pregnancy in a larger sample of women than in previous studies and, to our knowledge, for the first time in a population from the Mediterranean area. It provides valuable data on a wide range of FA, these being total SFA and lauric acid, myristic acid, palmitic acid and stearic acid; total MUFA and palmitoleic acid and oleic acid; total n-6 PUFA and linoleic acid, dihomo$\gamma$-linolenic acid and AA; n-3 PUFA and EPA and total DHA. The data on these FA were determined using high-quality methods and evaluated at two moments of gestation (first and third trimester). This has made it possible to describe changes to the different types of FA during pregnancy. The concentrations of SFA, MUFA and PUFA $n-6$ increased from the first to the third trimester of gestation, except for AA, the concentrations of which decrease. In contrast, n-3 PUFA and EPA concentrations decreased during pregnancy, while DHA concentrations remained stable. It appears that essential fatty acids DHA and $\mathrm{AA}$, which have a preferential placental transfer to the foetus, do not evolve in the same way as other FA during pregnancy.

There are few research projects that have analysed SFA and MUFA serum concentrations throughout pregnancy, conducted with Brazilian and Dutch pregnant woman [17-19, 21]. Our results are consistent with their findings, as they also found an increase in SFA and MUFA during pregnancy. This increase could be due to an increase in biosynthesis to cover requirements, since the rise in serum concentration may not be related to the small variation in the mothers' dietary intake [30,31], as our research group found in this sample of healthy pregnant women in relation to their intake during pregnancy [32]. The SFA may play a key role in supporting foetal membrane growth, as the SFA bio-magnification process could help to satisfy AA demands in foetal circulation and DHA in the brain [33]. However, the crucial role played by SFA has yet to be determined. An adequate concentration of MUFA is also fundamental during pregnancy because a non-optimal level has been associated with a high risk of preterm delivery and low weight at birth [33]. Oleic acid concentration could be a biomarker for a lack of essential fatty acid in the mother's diet, as has been shown in animal models [33]. In addition, it has been suggested that the consumption of monounsaturated fats could benefit DHA metabolism because they have a low amount of LA and thus do not interfere with endogenous conversion of $\alpha$-linolenic acid (ALA) 
Table 2 Fatty acid concentration of maternal serum during pregnancy period from the first trimester to third trimester

\begin{tabular}{|c|c|c|c|c|c|}
\hline Fatty acids ( $\mu \mathrm{mol} / \mathrm{L})$ & & $\begin{array}{l}\text { First Trimester (T1) } \\
\text { (Mean } \pm \text { SD) }\end{array}$ & $\begin{array}{l}\text { Third Trimester (T3) } \\
\text { (Mean } \pm \text { SD) }\end{array}$ & $\begin{array}{l}p \text {-value } \\
\text { between }^{(a-b)}\end{array}$ & $\begin{array}{l}\% \text { of variation } \\
\text { from } T 1 \text { to } T 3 \\
\text { (Mean } \pm \text { SD) }\end{array}$ \\
\hline \multicolumn{6}{|l|}{ SFA } \\
\hline Lauric acid (C12:0) & $n=444$ & $40.14 \pm 10.61$ & $60.19 \pm 28.26$ & $<0.0001$ & $56.33 \pm 76.07$ \\
\hline Myristic acid (C14:0) & $n=450$ & $118.16 \pm 49.07$ & $207.80 \pm 80.31$ & $<0.0001$ & $96.32 \pm 94.19$ \\
\hline Palmitic acid (C16:0) & $n=443$ & $2904.40 \pm 1403.47$ & $8511.32 \pm 4293.76$ & $<0.0001$ & $234.69 \pm 195.97$ \\
\hline Stearic acid (C18:0) & $n=451$ & $690.31 \pm 208.05$ & $808.08 \pm 202.10$ & $<0.0001$ & $25.99 \pm 44.26$ \\
\hline Total SFA & $n=444$ & $3765.31 \pm 1614.17$ & $9625.65 \pm 4574.33$ & $<0.0001$ & $184.93 \pm 154.49$ \\
\hline \multicolumn{6}{|l|}{ MUFA } \\
\hline Palmitoleic acid (C16:1n-7) & $n=444$ & $186.37 \pm 45.88$ & $256.66 \pm 89.61$ & $<0.0001$ & $42.94 \pm 54.20$ \\
\hline Oleic acid (C18:1n-9) & $n=441$ & $1444.74 \pm 461.90$ & $2843.85 \pm 1251.92$ & $<0.0001$ & $108.61 \pm 100.53$ \\
\hline Total MUFA & $n=442$ & $1634.54 \pm 500.21$ & $3116.39 \pm 1330.86$ & $<0.0001$ & $101.02 \pm 93.05$ \\
\hline \multicolumn{6}{|l|}{ n-6 PUFA } \\
\hline Linoleic acid (LA) (C18:2n-6) & $n=446$ & $3355.67 \pm 1230.19$ & $6321.14 \pm 2786.02$ & $<0.0001$ & $107.35 \pm 111.69$ \\
\hline Dihomo- $\gamma$-linolenic acid (DHGLA) (C20:3n-6) & $n=450$ & $229.99 \pm 90.64$ & $246.20 \pm 85.27$ & 0.001 & $19.38 \pm 53.87$ \\
\hline Arachidonic acid (AA) (C20:4n-6) & $n=451$ & $830.82 \pm 276.15$ & $722.63 \pm 220.05$ & $<0.0001$ & $-6.33 \pm 34.88$ \\
\hline Total n-6 PUFA & $n=444$ & $4433.77 \pm 1469.64$ & $7278.06 \pm 2919.04$ & $<0.0001$ & $77.85 \pm 85.91$ \\
\hline \multicolumn{6}{|l|}{ n-3 PUFA } \\
\hline Eicosapentaenoic acid (EPA) (C20:5n-3) & $n=446$ & $35.03 \pm 23.95$ & $23.88 \pm 16.93$ & $<0.0001$ & $-14.93 \pm 68.21$ \\
\hline Docosahexaenoic acid (DHA) (C22:6n-3) & $n=456$ & $240.28 \pm 73.47$ & $236.60 \pm 71.77$ & 0.357 & $4.89 \pm 37.7$ \\
\hline Total n-3 PUFA & $n=454$ & $276.98 \pm 94.07$ & $260.52 \pm 84.33$ & 0.001 & $1.21 \pm 38.34$ \\
\hline Total LCFA & $n=442$ & $10,073.15 \pm 3349.07$ & $20,480.82 \pm 8335.23$ & $<0.0001$ & $118.63 \pm 103.14$ \\
\hline
\end{tabular}

Fatty acid concentration ( $\mu \mathrm{mol} / \mathrm{L}$ ) of maternal serum and $\%$ of variation from the first to third trimester are presented as mean \pm standard deviation (SD). SFA, saturated fatty acids; MUFA, monounsaturated fatty acids; n-6 PUFA, omega-6 polyunsaturated fatty acids; n-3 PUFA, omega-3 polyunsaturated fatty acids. Total LCFA, long chain fatty acids $=$ total SFA + total MUFA + total $n-6$ PUFA + total $n-3$ MUFA

to DHA [34]. Further research is needed to analyse their role during pregnancy.

In terms of PUFA, we found that n-6 PUFA concentrations increased during gestation, whereas essential fatty acid (EPA, DHA, AA) concentrations decreased or remained stable. Our results are partially in concordance with the literature. For instance, Zhao et al. [20] observed that Canadian pregnant women without diabetes showed higher serum concentrations of all fatty acids except EPA at 35-37 weeks of pregnancy than at 24-28 weeks. A systematic review found that levels of absolute concentrations of DHA increased and EPA did not change [16]. Pinto et al. [17], in a study involving Brazilian pregnant women, showed that from the first to second trimesters the serum FA concentrations, EPA + DHA, increased greatly, but a slight increase in DHA and a decrease in EPA (although not statistically significant) were observed in the third trimester. However, our study found no significant variation in DHA from the first to third trimesters and observed a significant decrease of around 15\% in EPA concentrations. It may be that the DHA synthesis from EPA was more efficient than from ALA $[35,36]$ and that DHA concentrations could be regulated by a different biosynthesis or mobilization mechanism [35-38]. In fact, n-6 and n-3 PUFA families compete for metabolism by desaturation enzymes, and therefore a Western or industrialized diet rich in vegetable oils that are high in LA would inhibit the synthesis of n-3 PUFA by ALA. In addition, AA is an essential fatty acid that is preferential for transfer to the foetus along with DHA [1]. Our findings show that AA concentrations also decrease by around $8 \%$ during pregnancy. Although LA serum concentrations increased, it does not appear that it was to be converted into AA, which may suggest that enzymatic activity of delta 5 desaturase could be reduced. Other researchers have suggested that LA may compete with other fatty acids, including AA, for acylation [39, 40]. In agreement with our results, Stewart et al. [38] found no differences in the AA concentrations of erythrocytes; however, other researchers have found an increase in AA serum concentrations during pregnancy $[16,17,20]$. The reasons for the different findings are unclear. It should be noted that the systematic review by Wilson et al. [16] included only three studies that assessed serum concentrations of FA, one from Brazil and two from the Netherlands, whose populations have different dietary patterns with a high level of heterogeneity between them. It is possible that the differences could be due to when the 


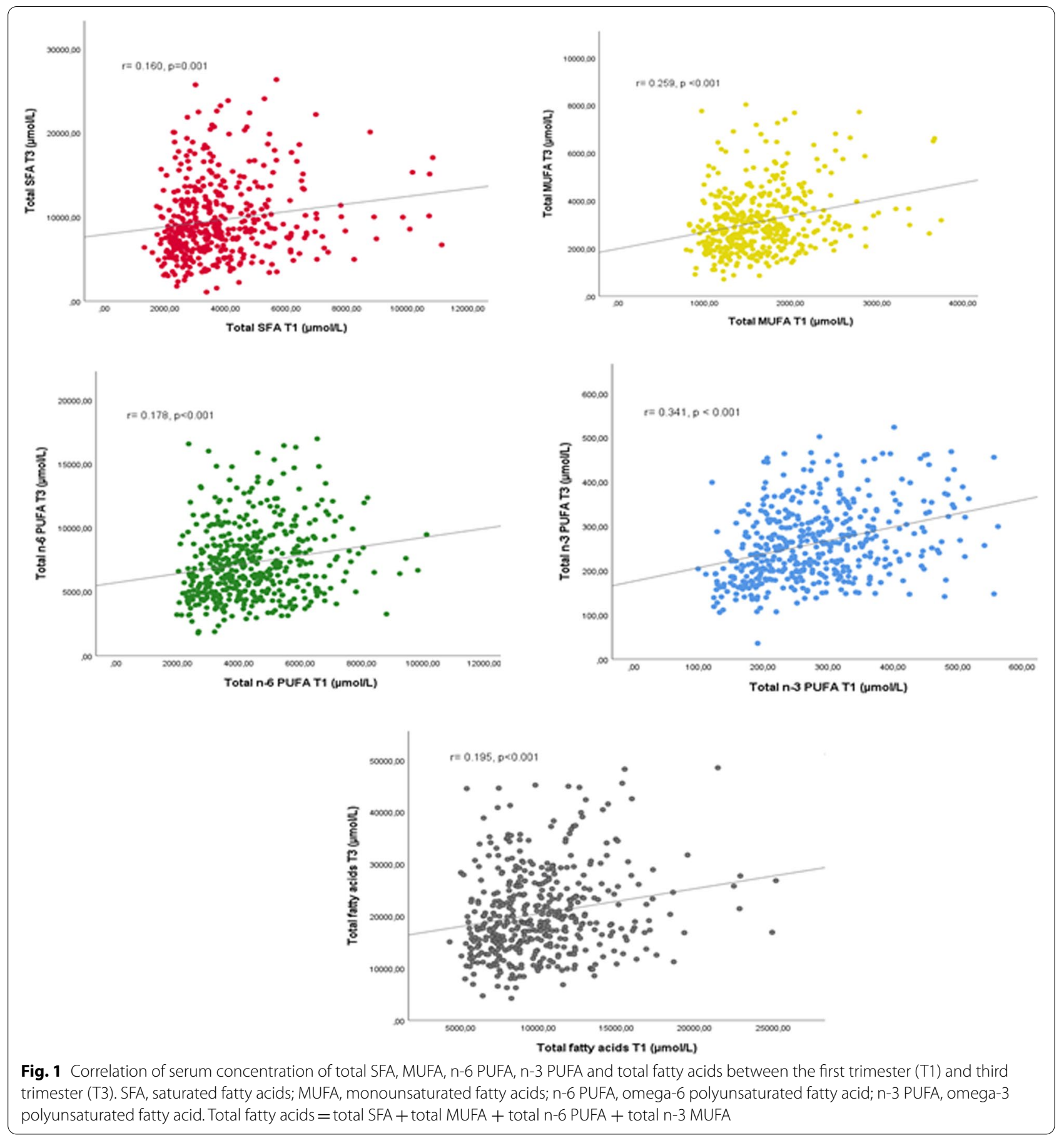

measurements were taken, to sample size, the fatty acid assay protocol or the study populations. More research is needed to clarify how essential fatty acids change over the course of the entire pregnancy.

Furthermore, our study is a secondary analysis of a clinical trial of iron supplementation. In this sense, a physiological relationship between iron and fatty acids is hypostatized since, for instance, ferritin may incorporate FA in its structure in addition to iron [41, 42]. However, our results did not show that the iron dosage of the supplementation had any effect on FA concentration.

To our knowledge this is the first study of the changes in maternal serum FA during pregnancy in a Mediterranean region. According our findings, circulating FA 
- specifically SFA, MUFA and n-6 PUFA - increases throughout pregnancy. This could be explained by a synthesis mechanism or mobilization of the maternal fat store, since the concentrations may not be related to dietary intake. Given this situation, we could consider that the increase in SFA, MUFA and n-6 PUFA serum concentration during pregnancy seems to indicate that the metabolic mechanisms involved may offer an optimal nutritional availability to meet the increased demands of these FA due to the increased growth and development of the foetus at the end of gestation. Nevertheless, essential fatty acid concentrations, which are preferential for transfer to the placenta, may be more complexly regulated. During early pregnancy the PUFA derived from the diet are stored in maternal adipose tissue. Indeed, n-3 PUFA serum concentration has been related to food consumption in pregnant woman $[8,10,17,27]$ insofar as it is transferred from intake to maternal fat stores. The amount of n-3 PUFA stored in the maternal adipose tissue could vary depending on the habitual dietary intake of n-3 fatty acids. However, metabolic and physiological changes occur during pregnancy, and these involve the complex synchronization of maternal, placental and foetal fat metabolism to ensure a continuous supply of $n-3$ and n-6 PUFA to the foetus [35]. Some mechanisms could be related to the mobilization of maternal fat stores, an increase in the elongation and desaturation of FA regulated by oestrogen, the differential placental uptake of FA or the rate of DHA conversion synchronized according to the period of maximal foetal demand [35-38]. The transfer of n-3 and n-6 PUFA to the foetus increases from 20 weeks of gestation and reaches a peak in the last trimester of pregnancy with a high maternal transfer of DHA and AA to ensure foetal demand $[1,35]$. Overall, this decrease or non-modification of essential fatty acids during pregnancy, closely related to their intake, indicates that there are specific physiological mechanisms, such as preferential FA transfer between mother and foetus, so as to ensure high foetal demands are met [35]. This might put the mother at risk of deficiency when her intake is insufficient. Indeed, it has been found that low n-3 PUFA status and imbalance between n- 6 and n-3 PUFA in early pregnancy increase the risk of postpartum depression after delivery [10, 14]. This could lead us to consider that the mechanisms involved in its storage in maternal fat, together with other possible complex mechanisms of metabolic synchronization of maternal, placental and foetal fat could be insufficient to guarantee the FA needs in situations of low contributions. In general, for all FA, further testing of normal FA levels during pregnancy would be necessary for better evaluations and follow-ups. In addition, more research is needed on the metabolic mechanisms of FA during pregnancy.
Given that essential fatty acid concentrations and their balance depend on both biological mechanisms and dietary supply, an optimal dietary intake is needed to ensure proper functioning of biological mechanisms so as to facilitate correct foetal growth and development. For this reason, we believe that the pregnancy control reviews carried out by obstetricians and health professionals should include dietary advice on foods rich in these FA, verify consumption of these foods and thus FA, and determine the serum concentrations of essential FA. There is some evidence that supports the benefits of an adequate intake of FA. Fish and seafood consumption during pregnancy has been reported to be beneficial for offspring neurocognitive development [43, 44]. Several other research projects have associated an inadequate balance of $n-3$ and n-6 PUFA profiles during pregnancy with deleterious consequences for the child, such as neurodevelopment disorder [43, 6], immune system disease $[45,46]$ and cardiometabolic risk and adiposity $[47,48]$. In addition, AA is a precursor of prostaglandin and other metabolites, which play a key role in the late pregnancy period and delivery [49]. Nevertheless, an excessive activation of inflammatory mediators during pregnancy might lead to deleterious effects, such as gestational diabetes, preeclampsia or pre-term delivery [15].

The main strength of our study is its longitudinal design, which enabled us to analyse variations in serum FA concentrations throughout pregnancy. Another strength is the bigger sample size compared to other research [17-21]. However, several limitations should be considered. Although the sample is of voluntary pregnant women from a randomized clinical trial with a control group, the sample avoids the selective survival error by selecting women from each area of the primary health care ASSIR service specific for pregnant women, from an entire region. This provided a good representation of the socioeconomic and educational levels of the sample, among other aspects. In addition, exhaustive inclusion and exclusion criteria were fulfilled, previously designed and agreed on with the ASSIR service, thus guaranteeing a sample of healthy pregnant adult women. Incentives were given to minimize the loss of women in the pregnancy follow-up, consisting of follow-up visits interspersed with study visits, economic incentives for midwives who carried out these visits, and informational incentives for mothers about their health status and that of their baby. In the design phase of the study, all procedures, masking and quality control were systematized and monitored by a group external to the research group. Nevertheless, our findings may not be extended to all populations as ethnicity, nutritional habits and lifestyle may impact on the fatty acid levels. In the assessment of FA, it was not possible to assess ALA, one of the main 
n-3 PUFA, in our sample. Furthermore, due to the lack of reference values, our study was unable to apply a cut-off value regarding the percentage of the pregnant population that is above or below the optimal level. Therefore, our findings indicate that it is necessary to establish reference values according to the variation of FA in each trimester. Moreover, taking into account that our results show a decrease in essential fatty acids at the end of pregnancy due to the demands of the foetus, health and nutrition counselling provided by obstetricians and health professionals could add that mothers consume foods rich in FA from the beginning of the pregnancy to prevent levels from dropping excessively at the end of gestation. In addition, clinical guidelines during pregnancy could include the assessment of FA in each trimester, so that professionals could identify those women who are at risk and recommend specific treatment or advice if necessary.

In conclusion, the circulating serum concentration of SFA, MUFA and n-6 PUFA increases during pregnancy, whereas essential fatty acids such as AA and EPA decrease and DHA remains unchanged. Therefore, the increase in SFA, MUFA and n-6 PUFA serum concentration during pregnancy, which is related to specific metabolic mechanisms during pregnancy, seems to offer an adequate availability of these FA for increased foetal growth and development at the end of pregnancy. However, the decrease or non-increase in essential fatty acids during pregnancy may indicate that there are specific physiological mechanisms that might put the mother at risk of deficiency when her intake is insufficient. More research is necessary to analyse the role of FA during pregnancy.

\footnotetext{
Abbreviations

FA: Fatty acids; SFA: Saturated fatty acids; MUFA: Monounsaturated fatty acids; PUFA: Polyunsaturated fatty acids; n-6 PUFA: Omega- 6 polyunsaturated fatty acids; n-3 PUFA: Omega-3 polyunsaturated fatty acids; DHA: Docosahexaenoic acid; EPA: Eicosapentaenoic acid; AA: Arachidonic acid; LA: Linoleic acid; ALA: a-linolenic acid.
}

\begin{abstract}
Acknowledgments
We thank the Jordi Gol Research Institute in Primary Care (Institut d'Investigació en Atenció Primària; IDIAP) for their guidance regarding ethical matters. We also thank the entities and participants in the ECLIPSES study: Research Group in Nutrition and Mental Health (NUTRISAM), Universitat Rovira i Virgili, Reus, Spain; Sexual and Reproductive Health Care Services (ASSIR) of Tarragona, Spain, and the team of midwives recruiting for the study; the research support Unit-Tarragona and Central Unit-Barcelona of the Institut d'Atenció Primària IDIAP Jordi Gol, Institut Català de la Salut; Laboratory of Institut Català de la Salut (ICS), University Hospital of Tarragona Joan XXIII, Tarragona, Spain. Finally, we thank the Centre for Omic Sciences (COS) Joint Unit of the Universitat Rovira i Virgili-Eurecat for their contribution to the chromatographic and mass spectrometry analysis.
\end{abstract}

\section{Authors' contributions}

VA conceptualized, designed, and coordinated the study. VA and JC conducted the research and research process. CM-G and EA analysed the data. EA, CM.-G, VA, CH-M, NV and JC contributed to the curation of data. EA, CM-G, VA prepared and wrote the original draft of the manuscript. EA, CM-G, VA, CH-M,
NV, JC contributed to writing, reviewing and editing the final manuscript. All authors have read and agreed to the published version of the manuscript.

\section{Funding}

The study was funded by a grant (PI17/01754) from the Health Research Fund of the Ministry of Health and Consumption (Madrid, Spain) (Instituto de Salud Carlos III, Fondo de Investigación Sanitaria, Ministerio de Sanidad y Consumo) and co-funded by the European Union (ERDF/ESF,"A way to make Europe"/"Investing in your future"). The funding bodies played no part in the design of the study, collection and interpretation of data, or decision to publish.

\section{Availability of data and materials}

The datasets used and/or analysed during the current study are only available from the corresponding author on reasonable request.

\section{Declarations}

\section{Ethics approval and consent to participate}

The study was conducted according to the guidelines of the Declaration of Helsinki, and approved by the Clinical Research Ethics Committee of the Jordi Gol Research Institute in Primary Care [Instituto de Investigación en Atención Primaria; IDIAP], the Pere Virgili Institute for Health Research (Instituto de investigación Pere Virgili; IISPV), and the Spanish Agency for Medicines and Health Products [Agencia Española del Medicamento y Productos Sanitarios; AEMPS] (AEMPS IJG-FER-2012 on Jul 9, 2018)."The study is registered in ClinicalTrials. gov with identification number NCT03196882 and in the EU Clinical Trial Register, EUCTR-2012-005480-28. All participants who agreed to participate signed the informed consent form.

\section{Consent for publication \\ Not applicable.}

\section{Competing interests}

The authors declare that they have no competing interests.

\section{Author details}

${ }^{1}$ Research Group on Nutrition and Mental Health (NUTRISAM), Universitat Rovira i Virgili, 43201 Reus, Spain. ${ }^{2}$ Institut d'Investigació Sanitària Pere Virgili (IISPV), 43003 Tarragona, Spain. ${ }^{3}$ Clinical Chemistry Laboratory, Catalan Institute of Health (ICS)-Camp de Tarragona-Terres de I'Ebre, Joan XXIII University Hospital in Tarragona, 43005 Tarragona, Spain. ${ }^{4}$ Research Center for Behavior Assessment (CRAMC), Universitat Rovira i Virgili, Tarragona, Spain.

Received: 14 June 2021 Accepted: 26 October 2021

Published online: 17 November 2021

\section{References}

1. Duttaroy A, Basak S. Maternal dietary fatty acids and their roles in human placental development. Prostaglandins Leukot Essent Fatty Acids. 2020;55:102080. https://doi.org/10.1016/j.plefa.2020.102080.

2. Birch EE, Carlson SE, Hoffman DR, Fitzgerald-Gustafson MK, Fu VLN, Drover JR, et al. The DIAMOND (DHA intake and measurement of neural development) study: a double-masked, randomized controlled clinical trial of the maturation of infant visual acuity as a function of the dietary level of docosahexaenoic acid. Am J Clin Nutr. 2010;91:848-59. https://doi.org/10.3945/ajcn.2009.28557.

3. Campoy C, Escolano-Margarit M, Anjos T, Szajewska H, Uauy R. Omega 3 fatty acids on child growth, visual acuity and neurodevelopment. Br J Nutr. 2012;107(Supple2):S85-S106. https://doi.org/10.1017/S0007 114512001493

4. Koletzko B, Lien E, Agostoni C, Böhles H, Campoy C, Cetin I, et al. The roles of long-chain polyunsaturated fatty acids in pregnancy, lactation and infancy: review of current knowledge and consensus recommendations. J Perinat Med. 2008;36:5-14 https://doi.org/10.1515/JPM.2008. 001. 
5. Lauritzen L, Brambilla P, Mazzocchi A, Harsløf LBS, Ciappolino V, Agoston C. DHA effects in brain development and function. Nutrients. 2016;8:6 https://doi.org/10.3390/nu8010006.

6. Steenweg-de Graaff J, Tiemeier H, Ghassabian A, Rijlaarsdam J, Jaddoe VWV, Frank $C$, et al. Maternal fatty acid status during pregnancy and child autistic traits: the generation R study. Am J Epidemiol. 2016;183:792-9 https://doi.org/10.1093/aje/kwv263.

7. Campoy C, Azaryah H, Torres-Espínola FJ, Martínez-Zaldívar C, GarcíaSantos JA, Demmelmair H, et al. Long-chain polyunsaturated fatty acids, Homocysteine at birth and fatty acid Desaturase gene cluster polymorphisms are associated with Children's processing speed up to age 9 years. Nutrients. 2020;13:131 https://doi.org/10.3390/nu13010131.

8. Hoge A, Bernardy F, Donneau AF, Dardenne N, Degée S, Timmermans M, et al. Low omega-3 index values and monounsaturated fatty acid levels in early pregnancy: an analysis of maternal erythrocytes fatty acids. Lipids Health Dis. 2018;17:1-11 https://doi.org/10.1186/s12944-018-0716-6.

9. Gellert S, Schuchardt JP, Hahn A. Higher omega-3 index and DHA status in pregnant women compared to lactating women — results from a German nation-wide cross-sectional study. Prostaglandins Leukot Essent Fatty Acids. 2016:109:22-8 https://doi.org/10.1016/j.plefa.2016.04.002

10. Markhus MW, Rasinger JD, Malde MK, Frøyland L, Skotheim S, Braarud HC, et al. Docosahexaenoic acid status in pregnancy determines the maternal Docosahexaenoic acid status 3-, 6- and 12 months postpartum. Results from a longitudinal observational study. PLoS One. 2015;10:e0136409 https://doi.org/10.1371/journal.pone.0136409.

11. Shrestha N, Sleep SL, Cuffe JSM, Holland OJ, Perkins AV, Yau SY, et al. Role of omega- 6 and omega-3 fatty acids in fetal programming. Clin Exp Pharmacol Physiol. 2020;47:907-15 https://doi.org/10.1111/1440-1681.13244.

12. Shrestha N, Holland OJ, Kent NL, Perkins AV, McAinch AJ, Cuffe JSM, et al. Maternal high linoleic acid alters placental fatty acid composition. Nutrients. 2020:12:2183 https://doi.org/10.3390/nu12082183.

13. Vaz JS, Kac G, Nardi AE, Hibbeln RJ. Omega- 6 fatty acids and greater likelihood of suicide risk and major depression in early pregnancy. J Affect Disord. 2014;152-154:76-82 https://doi.org/10.1016/j.jad.2013.04.045.

14. Hoge A, Tabar V, Donneau AF, Dardenne N, Degée S, Timmermans M, et al. Imbalance between omega- 6 and omega-3 polyunsaturated fatty acids in early pregnancy is predictive of postpartum depression in a Belgian cohort. Nutrients. 2019;11:1-14 https://doi.org/10.3390/nu110 40876.

15. Szczuko M, Kikut J, Komorniak N, Bilicki J, Celewicz Z, Ziętek M, et al. The role of Arachidonic and linoleic acid derivatives in pathological pregnancies and the human reproduction process. Int J Mol Sci. 2020;21:9628 https://doi.org/10.3390/ijms21249628.

16. Wilson NA, Mantzioris E, Middleton PT, Muhlhausler BS. Gestational age and maternal status of DHA and other polyunsaturated fatty acids in pregnancy: a systematic review. Prostaglandins Leukot Essent Fatty Acids. 2019;144:16-31 https://doi.org/10.1016/.j.plefa.2019.04.006.

17. Pinto TJ, Farias DR, Rebelo F, Lepsch J, Vaz JS, Moreira JD, et al. Lower interpartum interval and unhealthy life-style factors are inversely associated with $n-3$ essential fatty acids changes during pregnancy: a prospective cohort with Brazilian women. PLoS One. 2015;10:e0121151 https://doi. org/10.1371/journal.pone.0121151.

18. Al MD, van Houwelingen AC, Hornstra G. Relation between birth order and the maternal and neonatal docosahexaenoic acid status. Eur J Clin Nutr. 1997:51:548-53 https://doi.org/10.1038/s.ejcn.1600444.

19. Al MD, van Houwelingen AC, Kester AD, Hasaart THM, De Jong AEP, Hornstra G. Maternal essential fatty acid patterns during normal pregnancy and their relationship to the neonatal essential fatty acid status. Br J Nutr. 1995;74:55-68 https://doi.org/10.1079/BJN19950106.

20. Zhao JP, Levy E, Shatenstein B, Fraser WD, Julien P, Montoudis A, et al. Longitudinal circulating concentrations of long-chain polyunsaturated fatty acids in the third trimester of pregnancy in gestational diabetes. Diabet Med. 2016;33:939-46 https://doi.org/10.1111/dme.12978.

21. Otto SJ, Houwelingen AC, Antal M, Manninen A, Godfrey K, LópezJaramillo $\mathrm{P}$, et al. Maternal and neonatal essential fatty acid status in phospholipids: an international comparative study. Eur J Clin Nutr. 1997;51:232-42 https://doi.org/10.1038/sj.ejcn.1600390.

22. Arija V, Fargas F, March G, Abajo S, Basora J, Canals J, et al. Adapting iron dose supplementation in pregnancy for greater effectiveness on mother and child health: protocol of the ECLIPSES randomized clinical trial. BMC Pregnancy Child. 2014;14:33 https://doi.org/10.1186/1471-2393-14-33.
23. Iglesias I, Arija V, Aranda N, Aparicio E, Serrat N, Fragas F, et al. The effectiveness of different doses of Iron supplementation and the prenatal determinants of maternal Iron status in pregnant Spanish women: ECLIPSES study. Nutrients. 2019;11:2418 https://doi.org/10.3390/nu111 02418.

24. Institut d'Estadística de Catalunya. Classificació catalana d'ocupacions 2011 (CCO-201 1). [Institut d'Estadística de Catalunya]. Catalunya: Spain; 2011.

25. World Health Organization. Global Dabatase on Body Mass Index. 2006. http://apps.who.int/bmi/index.jsp.?introPage=intro_3.html. Accessed 18 Jan 2019.

26. David F, Tienpont B, Klee MS, Tripp P. Automated Sample Preparation for Profiling Fatty Acids in Blood and Plasma Using the Agilent 7693. Agil Appl Note [Internet]. 5990-4822E. 2009. Available from: https://www.agile nt.com/cs/library/applications/5990-4822EN.pdf Accessed 20 Jan 2021.

27. Aparicio E, Martín-Grau C, Bedmar C, Serrat N, Basora J, Arija V. Maternal factors associated with levels of fatty acids, specifically n-3 PUFA during pregnancy: ECLIPSES study. Nutrients. 2021;13:317 https://doi.org/10. 3390/nu13020317.

28. Mowbray FI, Fox-wasylyshyn SM, El-masri MM. Univariate outliers: a conceptual overview for the nurse researcher. Can I Nurs Res. 2019;51:31-7 https://doi.org/10.1177/0844562118786647.

29. Cousineau D, Chartier S. Outliers detection and treatment: a review. Int J Psychol Res. 2011;3:58-67 https://doi.org/10.21500/20112084.844.

30. Lepsch J, Vaz JS, Moreira JD, Pinto TJP, Soares-Mota M, Kac G. Food frequency questionnaire as an indicator of the serum composition of essential $n-3$ and $n-6$ polyunsaturated fatty acids in early pregnancy, according to body mass index. J Hum Nutr Diet. 2015;28:85-94 https:// doi.org/10.1111/jhn.12225.

31. Marchioni DM, de Oliveira MF, Carioca AAF, Miranda AAM, Carvalho AM, Oki E, et al. Plasma fatty acids: biomarkers of dietary intake? Nutrition. 2019;59:77-82 https://doi.org/10.1016/j.nut.2018.08.008.

32. Aparicio E, Jardí C, Bedmar C, Pallejà M, Basora J, Arija V. The Eclipses study group. Nutrient intake during pregnancy and post-partum: ECLIPSES study. Nutrients. 2020;12:1325 https://doi.org/10.3390/nu12051325.

33. Ogundipe E, Johnson MR, Wang Y, Crawford MA. Peri-conception maternal lipid profiles predict pregnancy outcomes. Prostaglandins Leukot Essent Fatty Acids. 2016;114:35-43 https://doi.org/10.1016/j.plefa.2016. 08.012 .

34. Gibson RA, Muhlhausler B, Makrides M. Conversion of linoleic acid and alpha-linolenic acid to long-chain polyunsaturated fatty acids (LCPUFAs), with a focus on pregnancy, lactation and the first 2 years of life. Matern Child Nutr. 2011;7(Suppl 2):17-26 https://doi.org/10.1111/j.1740-8709. 2011.00299.x.

35. Haggarty P. Fatty acid supply to the human fetus. Annu Rev Nutr. 2010;21:237-55 https://doi.org/10.1146/annurev.nutr.012809.104742.

36. Burdge GC, Wootton SA. Conversion of alpha-linolenic acid to eicosapentaenoic, docosapentaenoic and docosahexaenoic acids in young women. Br J Nutr. 2002;88:411-20 https://doi.org/10.1079/BJN2002689.

37. Dubé E, Gravel A, Martin C, Desparois G, Moussa I, Ethier-Chiasson M, et al. Modulation of fatty acid transport and metabolism by maternal obesity in the human full-term placenta. Biol Reprod. 2012;87(14):1-11 https://doi.org/10.1095/biolreprod.111.098095.

38. Stewart F, Rodie VA, Ramsay JE, Greer IA, Freeman DJ, Meyer BJ. Longitudinal assessment of erythrocyte fatty acid composition throughout pregnancy and post partum. Lipids. 2007;42:335-44 https://doi.org/10. 1007/s11745-006-3005-5.

39. Adam O, Wolfram G, Zöllner N. Influence of dietary linoleic acid intake with different fat intakes on arachidonic acid concentrations in plasma and platelet lipids and eicosanoid biosynthesis in female volunteers. Ann Nutr Metab. 2003;47:31-6 https://doi.org/10.1159/000068906.

40. Friesen RW, Innis SM. Linoleic acid is associated with lower long-chain n-6 and $\mathrm{n}-3$ fatty acids in red blood cell lipids of Canadian pregnant women. Am J Clin Nutr. 2010;91:23-31.

41. Zanzoni S, Pagano K, D'Onofrio M, Assfalg M, Ciambellotti S, Bernacchioni $C$, et al. Unsaturated long-chain fatty acids are preferred ferritin ligands that enhance Iron biomineralization. Chemistry. 2017;23:9879-87. https:// doi.org/10.1002/chem.201701164.

42. Bu W, Liu R, Cheung-Lau JC, Dmochowski IJ, Loll PJ, Eckenhoff RG. Ferritin couples iron and fatty acid metabolism. FASEB J. 2012;26:2394-400. https://doi.org/10.1096/f.11-198853. 
43. Hibbeln JR, Spiller P, Brenna JT, Golding J, Holub BJ, Harris WS, et al. Relationships between seafood consumption during pregnancy and childhood and neurocognitive development: two systematic reviews. Prostaglandins Leukot Essent Fatty Acids. 2019;151:14-36 https://doi.org/ 10.1016/j.plefa.2019.10.002.

44. Julvez J, Méndez M, Fernandez-Barres S, Romaguera D, Vioque J, Llop S, et al. Maternal consumption of seafood in pregnancy and child neuropsychological development: a longitudinal study based on a population with high consumption levels. Am J Epidemiol. 2016;183:169-82 https:// doi.org/10.1093/aje/kwv195.

45. Montes R, Chisaguano AM, Castellote ME, Sunyer J, López-Sabater MC. Fatty-acid composition of maternal and umbilical cord plasma and early childhood atopic eczema in a Spanish cohort. Eur J Clin Nutr. 2013;67:658-63 https://doi.org/10.1038/ejcn.2013.68.

46. Notenboom ML, Mommers M, Jansen EH, Penders J, Thijs C. Maternal fatty acid status in pregnancy and childhood atopic manifestations: KOALA birth cohort study. Clin Exp Allergy. 2011;41:407-16 https://doi. org/10.1111/j.1365-2222.2010.03672.x

47. Jochems SH, Gielen M, Rump P, Hornstra G, Zeegers MP. Potential programming of selected cardiometabolic risk factors at childhood by maternal polyunsaturated fatty acid availability in the MEFAB cohort. Prostaglandins Leukot Essent Fatty Acids. 2015;100:21-7 https://doi.org/ 10.1016/j.plefa.2015.06.001.

48. Vidakovic AJ, Jaddoe VW, Voortman T, Demmelmair H, Koletzko B, Gaillard R. Maternal plasma polyunsaturated fatty acid levels during pregnancy and childhood lipid and insulin levels. Nutr Metab Cardiovasc Dis. 2017;27:78-85 https://doi.org/10.1016/j.numecd.2016.10.001.

49. Kikut J, Komorniak N, Ziętek M, Palma J, Szczuko M. Inflammation with the participation of arachidonic (AA) and linoleic acid (LA) derivatives (HETEs and HODEs) is necessary in the course of a normal reproductive cycle and pregnancy. J Reprod Immunol. 2020;141:103177 https://doi. org/10.1016/j.jir.2020.103177.

\section{Publisher's Note}

Springer Nature remains neutral with regard to jurisdictional claims in published maps and institutional affiliations.

Ready to submit your research? Choose BMC and benefit from:

- fast, convenient online submission

- thorough peer review by experienced researchers in your field

- rapid publication on acceptance

- support for research data, including large and complex data types

- gold Open Access which fosters wider collaboration and increased citations

- maximum visibility for your research: over $100 \mathrm{M}$ website views per year

At BMC, research is always in progress.

Learn more biomedcentral.com/submissions 